\title{
The topology of the intergalactic medium from multiple lines of sight to quasars
}

\author{
S. Caucci ${ }^{1}$, P. Petitjean ${ }^{1}$, C. Pichon ${ }^{1}$, F. Stoehr ${ }^{1}$, \\ E. Rollinde ${ }^{1}$, S. Colombi ${ }^{1}$ and F. Coppolani ${ }^{2}$ \\ ${ }^{1}$ Institut d'Astrophysique de Paris - 98bis bd Arago, 75014 Paris, France \\ ${ }^{2}$ European Southern Observatory - Alonso de Córdova 3107, Vitacura, Santiago, Chile
}

\begin{abstract}
The spatial distribution of the gas in the intergalactic medium and its physical properties can be recovered thanks to the characteristics of the $\operatorname{Ly} \alpha$ absorption lines that are observed in the spectra of high redshift quasars, the so-called Ly $\alpha$ forest. We use inversion methods applied to a network of lines of sight to infer the topology of the intergalactic medium, based on reconstruction of the density field. The method has already been tested on simulated data.
\end{abstract}

\section{The Ly $\alpha$ forest}

It is well known that most of the absorption lines observed in the spectra of highredshift quasars are mainly produced by the neutral hydrogen (HI) present in the intergalactic medium (IGM), i.e. the gas located along the line of sight between us and the quasar. This part of the spectrum is the so-called Ly $\alpha$ forest. So, the analysis of the Ly $\alpha$ forest is a tool for recovering the physical properties of the IGM and its spatial distribution. Numerical simulations show that the distribution of the gas in the IGM follows the distribution of the dark matter, thus recovering the spatial distribution of the gas is a way to infer the distribution of the dark matter. In my PhD I reconstruct the spatial distribution of the IGM from a network of simulated lines of sight toward groups of quasars. In particular I characterise the topology of the reconstructed field and discuss what information can be derived about the density field of the gas from the reconstruction.

\section{Numerical simulations}

We used cosmological hydrodynamic simulations to study the global density field of the intergalactic medium. The simulations included both dark matter particles and a gaseous component. The dynamical evolution and the physical properties of the gas were calculated taking into account all the heating and cooling processes and the effects of an ionising UV background. We constructed simulated quasar absorption spectra along different lines of sight through the simulation box combining the effects of the neutral density, peculiar velocities, and thermal broadening. Comparison between observed and simulated spectra allows us to infer the properties of the gas that produces the absorption lines in real spectra, and in particular, its spatial distribution.

\section{Correlation functions}

For a pair of lines of sight we can define the flux correlation function in the following way:

$$
\xi(\theta, \Delta v)=\langle(F(\theta, \lambda+\Delta \lambda)-\langle F\rangle)(F(0, \lambda)-\langle F\rangle)\rangle_{\lambda},
$$


where $F$ is the flux along two lines of sight with separation $\theta$ and mean redshift $z$, and $\Delta \lambda=\lambda_{0}(1+z) \times \Delta v / c$, with $\lambda_{0}=\lambda_{\text {Ly } \alpha \text {, rest. }}$. For $\theta=0$ in particular, we obtain the longitudinal correlation function for a single line of sight, while for $\Delta v=0$ we obtain the so-called transverse correlation function. They give us information along the line of sight and perpendicular to the line of sight respectively. The study of the correlation functions has a two-fold importance:

- it allows us to recover the clustering properties of the IGM and the characteristic size of the structures responsible for the absorption;

- comparison between the transverse and the longitudinal correlation functions allows us to infer the geometry of the Universe, since they depend on the cosmological parameters in two different ways (see Alcock \& Paczyński 1979; Rollinde et al. 2003).

\subsection{Correlation in close pairs of quasars}

The correlation has been observed in close pairs of quasars. Both the longitudinal and the transverse observed correlations can be reproduced by using numerical simulations. Fig. 1

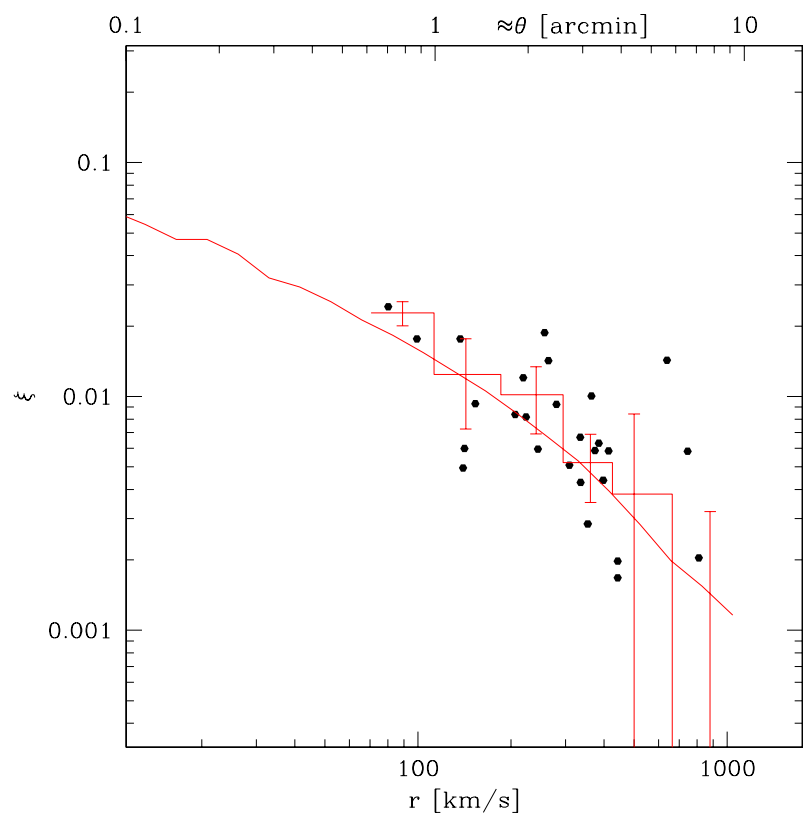

Figure 1. Black dots and histogram: observed transverse correlation function obtained for pairs of quasars. Solid line: transverse correlation function resulting from numerical simulation, calibrated in order to fit the longitudinal correlation function of the set of data.

(Coppolani et al. 2005 but see also Rollinde et al. 2003) shows the observed transverse correlation function (dots and histogram) obtained for a unique set of data, consisting of 32 pairs of QSOs observed with FORS at the VLT, with separations ranging from 0.6 to 9.5 arc-min. The solid line represents the transverse correlation function obtained from the numerical simulation. The cosmological parameters in the simulation have been chosen in order to reproduce the observed longitudinal correlation function, but with the same values of the parameters, we can also match the transverse correlation function. This fact shows that there is a good agreement between observations and simulations. The next step is to use numerical simulations to investigate the IGM density field in 3D. 


\section{Reconstruction of simulated fields}

By considering multiple lines of sight through the simulation, we can recover the 3D distribution of the IGM (Pichon et al. 2001). The results of different reconstructions

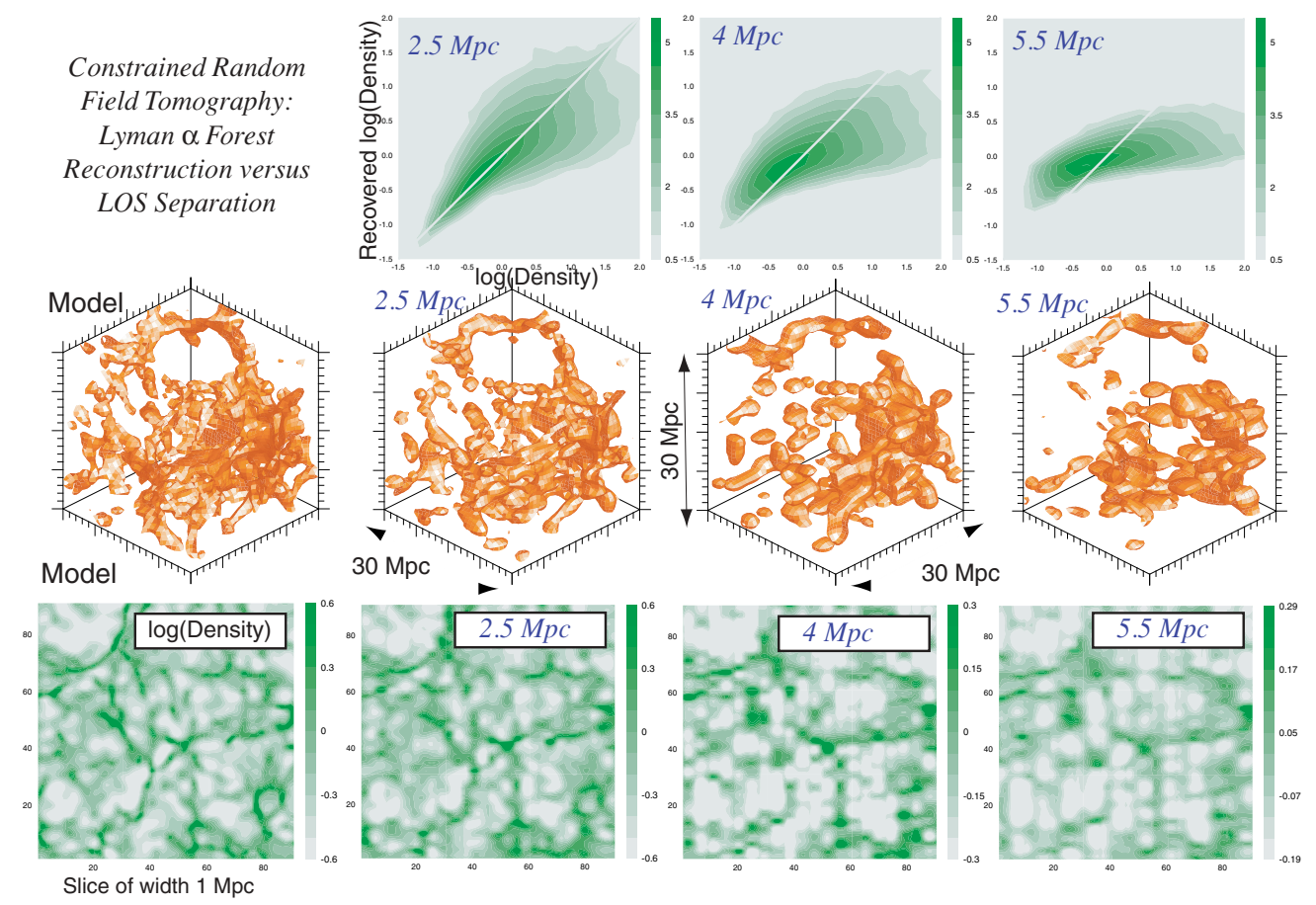

Figure 2. Reconstruction of the simulated density field from different sets of lines of sight. Middle row: 3D distribution of the IGM (left panel) and three reconstructions obtained for a network of lines of sight with constant separation (2.5, 4, and $5 \mathrm{Mpc}$ from left to right) in the simulation. Bottom row: slice of $1 \times 80 \times 80 \mathrm{Mpc}$ across the simulation (left panel) and the reconstructed fields. Top row: recovered density after reconstruction as a function of the density in the simulation.

are shown in Fig. 2. It can be seen that for a constant separation between the lines of sight the reconstruction is very good in the case of small separations $(2.5 \mathrm{Mpc})$. If we increase the separation (to 4 or $5.5 \mathrm{Mpc}$ ), information on the details of the density field is lost except for the general topology of the filamentary structures, which is globally recovered. In my $\mathrm{PhD}$ I consider more realistic situations mimicking groups of QSOs. I will reconstruct the $3 \mathrm{D}$ density field from different (not equally-spaced) lines of sight by means of the inversion method and characterise the topology of the reconstructed field, in order to understand what information can be derived from the reconstructed fields in realistic cases.

\section{References}

Alcock, C., Paczyński, B., 1979, Nature, 281, 358

Coppolani, F., Petitjean, P., Stoehr, F., Pichon, C., 2005, in preparation

Pichon, C., Vergely, J. L., Rollinde, E., Colombi, S., Petitjean, P., 2001, MNRAS, 326, 597

Rollinde, E., Petitjean, P., Pichon, C., Colombi, S., Aracil, B., D’Odorico, V., Haehnelt, M. G., 2003, MNRAS, 341, 1279 